\title{
Structure of the exact wave function
}

AUTHOR(S):

Nakatsuji, $\mathrm{H}$

CITATION:

Nakatsuji, $H$. Structure of the exact wave function. JOURNAL OF CHEMICAL PHYSICS 2000, 113(8): 2949-2956

ISSUE DATE:

2000-08-22

URL:

http://hdl.handle.net/2433/39727

\section{RIGHT:}

Copyright 2000 American Institute of Physics. This article may be downloaded for personal use only. Any other use requires prior permission of the author and the American Institute of Physics. 


\title{
Structure of the exact wave function
}

\author{
Hiroshi Nakatsuji \\ Department of Synthetic Chemistry and Biological Chemistry, Graduate School of Engineering, \\ Kyoto University, Kyoto 606, Japan
}

(Received 5 January 2000; accepted 31 May 2000)

\begin{abstract}
We investigate the structure of the exact wave function as a solution of the Schrödinger equation, aiming the singles and doubles description of the exact wave function. The basis is that the Hamiltonian involves only one and two body operators. We first present two theorems that indicate a possibility of the singles and doubles description of the exact wave function. We then examine the exponential ansatz, as this theorem implies it to be a possible structure of the exact wave function. Variational CCS (singles) wave function is shown to be certainly exact for one particle Hamiltonian. Thouless transformation plays an important role in the formulation. The conventional CCSD (singles and doubles) function is restrictive, even if it is solved variationally. A wider coupled cluster function with general singles and doubles substitution operators (CCGSD) is also not exact for the existence of noncommuting operators. We then analyze some formal properties of the full CI wave function, and finally, we propose an ansatz of the exact wave function and describe the method of solution. It involves successive solutions of the secular equations of the order of singles and doubles. It is variational and we can calculate both ground and excited states. (C) 2000 American Institute of Physics. [S0021-9606(00)30832-7]
\end{abstract}

\section{INTRODUCTION}

Solving the Schrödinger equation is not only a dream of a scientist but also has much practical utility. Exact predictions of chemical and physical properties and phenomena are our ultimate goal. Nowadays, only the full CI method is available for solving the exact wave function within a given basis set, but this method is too demanding computationally and therefore impractical even for a small system. The purpose of this paper is to search for the possibility of an easier way of solving the Schrödinger equation within singles and doubles. The basis is that the Hamiltonian involves only one and two particle operators.

We call a wave function $\psi$ to be "exact" when it satisfies the Schrödinger equation,

$$
(H-E) \psi=0 \text {, }
$$

where $H$ is the Hamiltonian of the system under consideration,

$$
H=\sum_{p r} v_{p}^{r} a_{r}^{+} a_{p}+\sum_{p q r s} w_{p q}^{r s} a_{r}^{+} a_{s}^{+} a_{q} a_{p}=H_{\mathrm{one}}+H_{\mathrm{two}},
$$

and $E$ is the energy of the system defined by

$$
\langle\psi|(H-E)| \psi\rangle=0 .
$$

The creation and annihilation operators, $a_{r}^{+}$and $a_{p}$, respectively, satisfy the anticommutation relation given by ${ }^{1}$

$$
\begin{aligned}
& a_{p}^{+} a_{q}+a_{q} a_{p}^{+}=\left[a_{p}^{+}, a_{q}\right]_{+}=\delta_{p q}, \\
& {\left[a_{p}^{+}, a_{q}^{+}\right]_{+}=0,} \\
& {\left[a_{p}, a_{q}\right]_{+}=0 .}
\end{aligned}
$$

The reference one-particle functions used for defining $a_{r}^{+}$ and $a_{p}$ are the Hartree-Fock (HF) orbitals in the HF wave function,

$$
|0\rangle=\left\|\ldots \ldots \varphi_{i} \ldots \ldots \varphi_{j} \ldots \ldots\right\| .
$$

We use the indices $i, j, k, l$ for occupied orbitals, $a, b, c, d$ for unoccupied orbitals, and $p, q, r, s$ for general orbitals. Therefore,

$$
\begin{aligned}
& a_{i}^{+}|0\rangle=0, \\
& a_{a}|0\rangle=0 .
\end{aligned}
$$

A point quite important to be mentioned here is that the Hamiltonian is composed of only one and two particle operators. There are no elementary physical operators that involve more-than-three body interactions. Because of this simplicity, we may imagine that the exact wave function should have some simple structure, even though it is not known now, because it is an eigenfunction of such a simple operator, Hamiltonian. This is a motivation of this research. In the formulation given below, we utilize only this fact, and therefore, the argument of this paper is valid to any kind of physical systems.

It is well known that the second order density matrix $\Gamma^{(2)}\left(1^{\prime} 2^{\prime} \mid 12\right)$ is enough to calculate all the elementary 
physical properties. ${ }^{2-4}$ We have the variational method for the direct determination of $\Gamma^{(2)}{ }^{5,6} \mathrm{We}$ also have the density equation, including only density matrices as variables, that is equivalent to the Schrödinger equation. ${ }^{7,8}$ These methods are based on the possibility of the singles and doubles description of quantum mechanics. The obstacle there is the N-representability, ${ }^{9}$ but much progress has been made recently along the density equation theory (DET) ${ }^{7-14}$ A review of the DET in chemical physics has recently been summarized by the present author. ${ }^{14}$

We now define "necessity" and "sufficiency" relations to the Schrödinger equation. If the "exact" wave function $\psi$, which is a solution of the Schrödinger equation, satisfies some equation, then that equation is called a necessary condition. On the other hand, if a $\psi$ satisfying some equation should always satisfy the Schrödinger equation, then this equation is called a sufficient condition of the Schrödinger equation. When some equation is not only necessary but also sufficient, then that equation is called to be "equivalent" to the Schrödinger equation. As such equivalent formula, we have variational principle, density equation, ${ }^{7,8}$ etc. The equivalent equation has the same determinative power as the Schrödinger equation has, when it is solved appropriately. There are many necessary (but not sufficient) conditions of the Schrödinger equation, for example, Hellmann-Feynman theorem, virial theorem, etc., but generally speaking, the determinative power of such an equation is limited. ${ }^{15}$

In this paper, the following equation plays an important role for judging a wave function $\psi$ to be exact, namely,

$$
\left\langle\psi\left|(H-E)^{2}\right| \psi\right\rangle=0,
$$

where the energy $E$ is defined by Eq. (1.3). This equation is equivalent to the Schrödinger equation ${ }^{16}$ in the necessary and sufficient sense and valid to both ground and excited states.

In Sec. II, we show the basic theorems that imply a possibility of the singles and doubles description of the exact wave function. As the theorem implies the exponential ansatz combined with the variational principle as a possible structure of the exact wave function, we devote Secs. III, IV, and $\mathrm{V}$ to the examinations of the exponential ansatz including singles and doubles excitation operators as a candidate of the exact wave function. Sec. VI gives an examination of the full CI wave function in a light of the present consideration, and in Sec. VII we give a proposal of the singles and doubles description of the exact wave function. Conclusions are given in Sec. VIII.

\section{BASIC THEOREM}

The theorem given below is very simple but its implication is important.

Theorem II- 1 . The wave function $\psi$ that satisfies the following two equations:

$$
\left\langle\psi\left|(H-E) a_{r}^{+} a_{p}\right| \psi\right\rangle=0
$$

and

$$
\left\langle\psi\left|(H-E) a_{r}^{+} a_{s}^{+} a_{q} a_{p}\right| \psi\right\rangle=0,
$$

for all the indices $p, q, r, s$ is sufficiently exact. These equations are also a necessary condition for the $\psi$ to be exact.
TABLE I. Single substitution operators. ${ }^{\mathrm{a}}$

\begin{tabular}{ccc}
\hline \hline & 1 & 2 \\
\hline $1^{\prime}$ & $a_{a}^{+} a_{i}$ & $a_{b}^{+} a_{a}$ \\
$2^{\prime}$ & $a_{j}^{+} a_{i}$ & $a_{i}^{+} a_{a}$ \\
\hline \hline
\end{tabular}

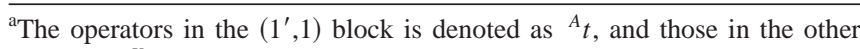
blocks as ${ }^{X} t$.

Proof. The necessity is trivial because the solution of the Schrödinger equation automatically satisfies Eqs. (2.1) and (2.2). The sufficiency is also easily shown. We calculate the following quantity using the definition of the Hamiltonian [Eq. (1.2)] and Eqs. (2.1) and (2.2), and show it to vanish identically

$$
\begin{aligned}
\langle\psi|(H-E) H| \psi\rangle= & \sum_{p r} v_{p}^{r}\left\langle\psi\left|(H-E) a_{r}^{+} a_{p}\right| \psi\right\rangle \\
& +\sum_{p q r s} w_{p q}^{r s}\left\langle\psi\left|(H-E) a_{r}^{+} a_{s}^{+} a_{q} a_{p}\right| \psi\right\rangle=0 .
\end{aligned}
$$

This equation combined with Eq. (1.3) gives Eq. (1.7), so that $\psi$ is exact. Thus, the necessary and sufficient theorem II-1 is proved. (QED)

The indices $p, q, r, s$ run both occupied and unoccupied orbitals, so that the single and double substitution operators appearing in Eqs. (2.1) and (2.2) are grouped as shown in Tables I and II. These operators are classified into two types, ${ }^{A} t$ and ${ }^{X} t$. The ${ }^{A} t$ operators are the ones in the $\left(1^{\prime}, 1\right)$ blocks of Tables I and II and involve only the excitation operators from occupied to unoccupied orbitals. The other operators are called ${ }^{X} t$ operators and have at least one creation or annihilation operator of the type $a_{i}^{+}$or $a_{a}$. The number of Eqs. (2.1) and (2.2) is

$$
M=m^{2}+\left[\frac{m}{2}(m-1)\right]^{2}
$$

with $m$ being the number of the active orbitals. It is larger than the number of the so-called singles and doubles, which is the number of the ${ }^{A} t$ type operators, but it is essentially in the same order. The equivalence of this set of equations with the Schrödinger equation implies an existence of the singles and doubles description of the exact wave function.

We note that even if we have the relation

$$
\left\langle\psi\left|(H-E) a_{s}^{+} a_{t}^{+} a_{u}^{+} a_{r} a_{q} a_{p}\right| \psi\right\rangle=0
$$

TABLE II. Double substitution operators. ${ }^{a}$

\begin{tabular}{ccccc}
\hline \hline & 1 & 2 & 3 & 4 \\
\hline $1^{\prime}$ & $a_{a}^{+} a_{c}^{+} a_{k} a_{i}$ & $a_{a}^{+} a_{k}^{+} a_{l} a_{i}$ & $a_{a}^{+} a_{d}^{+} a_{c} a_{i}$ & $a_{a}^{+} a_{k}^{+} a_{c} a_{i}$ \\
$2^{\prime}$ & $a_{j}^{+} a_{c}^{+} a_{k} a_{i}$ & $a_{j}^{+} a_{k}^{+} a_{l} a_{i}$ & $a_{j}^{+} a_{d}^{+} a_{c} a_{i}$ & $a_{j}^{+} a_{k}^{+} a_{c} a_{i}$ \\
$3^{\prime}$ & $a_{b}^{+} a_{c}^{+} a_{k} a_{a}$ & $a_{b}^{+} a_{k}^{+} a_{l} a_{a}$ & $a_{b}^{+} a_{d}^{+} a_{c} a_{a}$ & $a_{b}^{+} a_{k}^{+} a_{c} a_{a}$ \\
$4^{\prime}$ & $a_{i}^{+} a_{c}^{+} a_{k} a_{a}$ & $a_{i}^{+} a_{k}^{+} a_{l} a_{a}$ & $a_{i}^{+} a_{d}^{+} a_{c} a_{a}$ & $a_{i}^{+} a_{k}^{+} a_{c} a_{a}$ \\
\hline \hline
\end{tabular}

${ }^{a}$ The operators in the $\left(1^{\prime}, 1\right)$ block is denoted as ${ }^{A} t$, and those in the other blocks as ${ }^{X} t$. 
for the triple substitution operators and similar ones for higher operators, they have nothing to do with the derivation of Eq. (2.3), namely, with the proof of Theorem II-1. This again supports the existence of the singles and doubles description of the exact wave function. In a separate paper, ${ }^{17}$ we examined the roles of the higher excitations in the conventional configuration interaction $(\mathrm{CI})$ and coupled cluster (CC) wave functions.

Now, let us recall the variational principle of the form,

$$
\langle\psi|(H-E)| \delta \psi\rangle=0,
$$

which is equivalent to the Schrödinger equation in the necessary and sufficient sense. Comparing Eq. (2.6) with the equations in Theorem II-1, we can imagine the structure of the exact wave function as expressed by the following theorem.

Theorem II-2. Let us assume a wave function $\psi$ that has variables of the order of only singles and doubles,

$$
\psi=\psi\left(C_{p}^{r} a_{r}^{+} a_{p}, \quad C_{p q}^{r s} a_{r}^{+} a_{s}^{+} a_{q} a_{p}, \Phi_{K}\right),
$$

where $\Phi_{K}$ are the given reference functions, and further assume that $\psi$ satisfies

$$
\frac{\partial \psi}{\partial C_{p}^{r}}=a_{r}^{+} a_{p} \psi
$$

and

$$
\frac{\partial \psi}{\partial C_{p q}^{r s}}=a_{r}^{+} a_{s}^{+} a_{q} a_{p} \psi
$$

for the variations in the coefficients $C_{p}^{r}$ and $C_{p q}^{r s}$, respectively, then $\psi$ is exact.

Proof. Applying the variational principle, Eq. (2.6) under the assumption that the variation is done only for the unknown variables $C_{p}^{r}$ and $C_{p q}^{r s}$, then we get Eqs. (2.1) and (2.2) of Theorem II-1 from Eqs. (2.8) and (2.9), respectively. Therefore, $\psi$ is exact. (QED)

The numbers of Eqs. (2.8) and (2.9) are just the same as those of the unknown variables $C_{p}^{r}$ and $C_{p q}^{r s}$, respectively, included in the wave function of Eq. (2.7), so that we can determine all these unknown variables.

Theorem II-2 states that a sufficient condition for the $\psi$ to be exact is that it has the structure defined by Eqs. (2.8) and (2.9). The number of the variables is $M$ given by Eq. (2.4). $M$ is independent of the number of the electrons, $N$, of the system and is much smaller than that of the full CI, for which the number of variables is given by (for singlet)

$$
M_{\text {full - CI }}=\frac{1}{m+1}\left(\begin{array}{c}
m+1 \\
\frac{1}{2} N
\end{array}\right)\left(\begin{array}{c}
m+1 \\
\frac{1}{2} N+1
\end{array}\right),
$$

where () denotes binomial coefficient. This number depends not only on $m$ but also on $N$ and soon becomes intractably large even for relatively small systems.

Theorem II-2 is a sufficiency theorem and it does not claim the necessity. This means that the space of $\psi$ defined by Theorem II-2 may be smaller than the real space of the exact wave function. As expected from the argument on Eq.
(2.5), we note that even if we include triple, quadruple, etc., substitutions in our wave function and determine the associated variables by, e.g.,

$$
\frac{\partial \psi}{\partial C_{p q r}^{s t u}}=a_{s}^{+} a_{t}^{+} a_{u}^{+} a_{r} a_{q} a_{p} \psi
$$

they have nothing to do with the proof of the above theorem.

Since the wave function defined by the above theorem is exact, it should automatically satisfy several important properties, e.g., size-consistency and size-extesivity, ${ }^{18}$ upperbound nature for the ground state, the bound-from-below nature for the excited states, and a correct behavior in the homolytic bond fission process (multireference type nature of the wave function ${ }^{19}$ ).

From Eqs. (2.8) and (2.9), one may expect that the exponential ansatz of the wave function combined with the variational principle may represent the structure of the exact wave function. We therefore examine this possibility in the following three sections. We note that the variational derivation of the cluster expansion was given by the present author in the formulation of the symmetry adapted cluster (SAC) expansion. ${ }^{20}$ The SAC theory not only gives an accurate ground-state wave function, but also generates a set of excited functions that span the excited states. ${ }^{21}$ The SAC-CI theory utilizes such functions for describing excited states, ionized states, and electron-attached states. ${ }^{21-23}$ Kutzelnigg examined several different $\mathrm{CC}$ theories. ${ }^{24}$

\section{VARIATIONAL CCS FOR THE ONE-PARTICLE HAMILTONIAN}

As a simple prototype system, we consider here the system that has only one-particle term in the Hamiltonian,

$$
H=\sum_{p r} v_{p}^{r} a_{r}^{+} a_{p} .
$$

The HF model Hamiltonian is an example of this system. For such system, we have the following theorem:

Theorem III. For the one-particle Hamiltonian, the variational CCS wave function is exact.

Proof. The CCS wave function is defined by

$$
\begin{aligned}
\psi & =\exp (T)|0\rangle, \\
T & =\sum_{i, a} C_{i}^{a} a_{a}^{+} a_{i} .
\end{aligned}
$$

Since this wave function involves only commutable operators, we get

$$
\frac{\partial \psi}{\partial C_{i}^{a}}=a_{a}^{+} a_{i} \psi
$$

From the variational principle (Eq. (2.5)), we have

$$
\left\langle\psi\left|(H-E) a_{a}^{+} a_{i}\right| \psi\right\rangle=0,
$$

and the energy $E$ is defined by

$$
\langle\psi|(H-E)| \psi\rangle=0 .
$$


Since Eq. (3.4) is only for the ${ }^{A} t$ type operators, we have to formulate similar equations for the ${ }^{X} t$ type operators. For this purpose, we examine the effect of $a_{k}^{+}$and $a_{a}$ applied to $\psi$. First, we obtain

$$
a_{k}^{+} \exp (T)=\exp (T) a_{k}^{+}-\left(\sum_{a} C_{k}^{a} a_{a}^{+}\right) \exp (T)
$$

and

$$
a_{a} \exp (T)=\exp (T) a_{a}+\left(\sum_{i} C_{i}^{a} a_{i}\right) \exp (T),
$$

from the commutation rule given by Eq. (1.4) (see also the Appendix). Applying these equations to $|0\rangle$, we have

$$
\left(a_{k}^{+}+\sum_{a} C_{k}^{a} a_{a}^{+}\right) \psi=0
$$

and

$$
\left(a_{a}-\sum_{i} C_{i}^{a} a_{i}\right) \psi=0,
$$

and then we have

$$
\begin{aligned}
& a_{b}^{+} a_{a} \psi=\sum_{i} C_{i}^{a} a_{b}^{+} a_{i} \psi, \\
& a_{j}^{+} a_{i} \psi=\left(\delta_{i j}-\sum_{a} C_{j}^{a} a_{a}^{+} a_{i}\right) \psi,
\end{aligned}
$$

and

$$
a_{k}^{+} a_{b} \psi=\sum_{i} C_{i}^{b}\left(\delta_{i k}-\sum_{a} C_{k}^{a} a_{a}^{+} a_{i}\right) \psi .
$$

Now we calculate the following quantity, which is rewritten using Table I:

$$
\begin{aligned}
\langle\psi|(H-E) H| \psi\rangle= & \sum_{i, a} v_{i}^{a}\left\langle\psi\left|(H-E) a_{a}^{+} a_{i}\right| \psi\right\rangle \\
& +\sum_{a, b} v_{b}^{a}\left\langle\psi\left|(H-E) a_{a}^{+} a_{b}\right| \psi\right\rangle \\
& +\sum_{i, j} v_{i}^{j}\left\langle\psi\left|(H-E) a_{j}^{+} a_{i}\right| \psi\right\rangle \\
& +\sum_{a, i} v_{a}^{i}\left\langle\psi\left|(H-E) a_{i}^{+} a_{a}\right| \psi\right\rangle .
\end{aligned}
$$

The first term on the right-hand side vanishes identically by Eq. (3.4). The following three terms are also shown to vanish by using first Eqs. (3.10)-(3.12) and then Eqs. (3.4) and (3.5). Thus, we have shown that Eq.(1.7) holds for the CCS wave function; the CCS wave function is exact for the oneparticle Hamiltonian. (QED)

It is remarkable that in the CCS case, the substitution operators including the ${ }^{X} t$ type operators are all transformed to the terms including only the ${ }^{A} t$ type operators, and therefore, the last three terms of Eq. (3.13) become zero. This remarkable relation is derived from Eqs. (3.8) and (3.9), which are essentially equivalent to the Thouless theorem ${ }^{25}$ for the single-determinantal wave function.
Let us suppose the orbital transformations given by

$$
\chi_{i}=\varphi_{i}+\sum_{a} C_{i}^{a} \varphi_{a}
$$

and

$$
\chi_{a}=\varphi_{a}-\sum_{i} C_{i}^{a} \varphi_{i}
$$

then, the new set of creation and annihilation operators (denoted with prime) associated with the orbitals $\left\{\chi_{p}\right\}$ are given by

$$
a_{i^{\prime}}^{+}=a_{i}^{+}+\sum_{a} C_{i}^{a} a_{a}^{+}
$$

and

$$
a_{a^{\prime}}=a_{a}-\sum_{i} C_{i}^{a} a_{i}
$$

The CCS wave function given by Eq. (3.2) is nothing else but the Thouless transformation of the single determinant from $|0\rangle=\left\|\ldots \varphi_{i} \ldots.\right\|$ to $|\psi\rangle=\left\|\ldots \chi_{i} \ldots ..\right\|$, except for a normalization factor, and Eqs. (3.8) and (3.9) are nothing else but

$$
a_{i^{\prime}}^{+}\left\|\ldots \chi_{i} \ldots \ldots\right\|=0
$$

and

$$
a_{a^{\prime}}\left\|\ldots \chi_{i} \ldots . .\right\|=0,
$$

respectively. The corresponding equation for the original orbitals $\left\{\varphi_{p}\right\}$ is given by Eq. (1.6).

\section{VARIATIONAL CCSD}

Now, we come back to the general Hamiltonian given by Eq. (1.2) and consider how good is the CCSD wave function,

$$
\begin{aligned}
\psi & =\exp (T)|0\rangle, \\
T & =\sum_{i, a} C_{i}^{a} a_{a}^{+} a_{i}+\sum_{i j, a b} C_{i j}^{a b} a_{a}^{+} a_{b}^{+} a_{j} a_{i} .
\end{aligned}
$$

Applying the variational principle to the CCSD wave function, we get

$$
\left\langle\psi\left|(H-E) a_{a}^{+} a_{i}\right| \psi\right\rangle=0
$$

and

$$
\left\langle\psi\left|(H-E) a_{a}^{+} a_{b}^{+} a_{j} a_{i}\right| \psi\right\rangle=0 .
$$

We here want to examine the quantity,

$$
\begin{aligned}
\langle\psi|(H-E) H| \psi\rangle= & \sum_{p r} v_{p}^{r}\left\langle\psi\left|(H-E) a_{r}^{+} a_{p}\right| \psi\right\rangle \\
& +\sum_{p q r s} w_{p q}^{r s}\left\langle\psi\left|(H-E) a_{r}^{+} a_{s}^{+} a_{q} a_{p}\right| \psi\right\rangle,
\end{aligned}
$$

as in the previous section. The one and two electron operators of Eq. (4.4) run over all the elements $\left({ }^{A} t\right.$ and $\left.{ }^{X} t\right)$ of Tables I and II, respectively, while the operators in Eqs. (4.2) 
and (4.3) run only within the $\left(1^{\prime}, 1\right)$ blocks $\left({ }^{A} t\right.$ alone). The operators ${ }^{X} t$ are characterized by the inclusion of at least one $a_{i}^{+}$or $a_{a}$ operators.

We show in the Appendix the following relations:

$$
\left(a_{i}^{+}+\sum_{a} C_{i}^{a} a_{a}^{+}-2 \sum_{j a b} C_{j i}^{a b} a_{a}^{+} a_{b}^{+} a_{j}\right) \psi=0
$$

and

$$
\left(a_{a}-\sum_{i} C_{i}^{a} a_{i}+2 \sum_{i j b} C_{i j}^{b a} a_{b}^{+} a_{j} a_{i}\right) \psi=0 .
$$

In contrast to Eqs. (3.8) and (3.9) for the CCS wave function for the one-particle Hamiltonian, the above equations involve the products of three operators in the last terms; they originate from the double excitation terms in $T$ and complicate the Thouless transformation.

Equations (4.5) and (4.6) are utilized to transform the terms $a_{i}^{+} \psi$ and $a_{a} \psi$, respectively, involved in the term ${ }^{X} t \psi$ into the terms involving only the commutable operators of $a_{i}$ and $a_{a}^{+}$types. The $\left(1^{\prime}, 2\right)$ and $\left(2^{\prime}, 1\right)$ elements of the single substitution operators of Table I applied to $\psi$ are transformed as

$$
a_{c}^{+} a_{a} \psi=\left(\sum_{i} C_{i}^{a} a_{c}^{+} a_{i}-2 \sum_{i j b} C_{i j}^{b a} a_{c}^{+} a_{b}^{+} a_{j} a_{i}\right) \psi,
$$

and

$$
a_{i}^{+} a_{k} \psi=\left(\delta_{i k}-\sum_{a} C_{i}^{a} a_{a}^{+} a_{k}+2 \sum_{j a b} C_{j i}^{a b} a_{a}^{+} a_{b}^{+} a_{j} a_{k}\right) \psi .
$$

Therefore, from the variational condition given by Eqs. (4.2) and (4.3), we have

$$
\left\langle\psi\left|(H-E) a_{c}^{+} a_{a}\right| \psi\right\rangle=0,
$$

and

$$
\left\langle\psi\left|(H-E) a_{i}^{+} a_{k}\right| \psi\right\rangle=0,
$$

for the $\left(1^{\prime}, 2\right)$ and $\left(2^{\prime}, 1\right)$ elements of the single excitation operators. Eqs. (4.9) and (4.10) are very special among the $x_{t}$ type operators of Tables I and II. For example, the $\left(2^{\prime}, 2\right)$ element of the single substitution operator, $a_{i}^{+} a_{a}$, does not satisfy such a relation because the transformation based on Eqs. (4.5) and (4.6) leads to the terms involving three-particle excitations.

The ${ }^{X} t$ type double substitution operators shown in Table II applied to $\psi$ are similarly transformed into the terms that involve the excitations higher than triples. For example, the $\left(3^{\prime}, 1\right)$ element of Table II applied to $\psi$ is transformed as

$$
\begin{aligned}
& a_{c}^{+} a_{d}^{+} a_{k} a_{a} \psi \\
& \quad=\left(\sum_{i} C_{i}^{a} a_{c}^{+} a_{d}^{+} a_{k} a_{i}+2 \sum_{i j b} C_{i j}^{b a} a_{c}^{+} a_{d}^{+} a_{b}^{+} a_{k} a_{j} a_{i}\right) \psi
\end{aligned}
$$

that involves not only doubles but also triples, and the latter ones do not vanish by the variational conditions given by Eqs. (4.2) and (4.3). It is easy to show that the term ${ }^{X} t \psi$ with ${ }^{x} t$ given by Table II is transformed into the terms in-
TABLE III. Single substitution operators of Table I applied to $|0\rangle$.

\begin{tabular}{ccc}
\hline \hline & 1 & 2 \\
\hline $1^{\prime}$ & $a_{a}^{+} a_{i}|0\rangle$ & 0 \\
$2^{\prime}$ & $\delta_{i j}|0\rangle$ & 0 \\
\hline \hline
\end{tabular}

volving up to six-particle excitations. Actually, when ${ }^{x_{t}}$ involves $a_{i}^{+}$or $a_{a}$ operators $n$ times $(1 \leqslant n \leqslant 4)$, the highest excitation terms in ${ }^{X_{t}} \psi$ are $(n+2)$-particle ones. Therefore, for such ${ }^{X} t$ operators, we can not have the relations like Eqs. (4.9) and (4.10), so that the integral given by Eq. (4.4) does not vanish for the CCSD wave function. Thus, the conventional CCSD wave function is too restrictive to be an exact wave function, even if it is solved variationally. In other words, it does not have the freedom associated with the substitution operators expressed by ${ }_{t}$.

\section{COUPLED CLUSTER WITH THE GENERAL T-OPERATOR}

Based on the result of the preceding section, a natural next step is to examine the coupled cluster with more general $T$-operator. From the preceding argument, no restriction was there on the reference function, so that the simplest choice is the HF reference function $|0\rangle$. Then, we consider the cluster expansion with general $T$-operator as given by

$$
\begin{aligned}
\psi= & \exp (T)|0\rangle, \\
T= & \sum_{p r} C_{p}^{r} a_{r}^{+} a_{p}+\sum_{p q r s} C_{p q}^{r s} a_{r}^{+} a_{s}^{+} a_{q} a_{p} \\
& +\sum_{p q r s t u} C_{p q r}^{s t u} a_{s}^{+} a_{t}^{+} a_{u}^{+} a_{r} a_{q} a_{p}+\cdots
\end{aligned}
$$

In particular, we are interested in the following $T$-operator of singles and doubles:

$$
T=\sum_{p r} C_{p}^{r} a_{r}^{+} a_{p}+\sum_{p q r s} C_{p q}^{r s} a_{r}^{+} a_{s}^{+} a_{q} a_{p} .
$$

We refer to the coupled cluster with this general singles and doubles operators as CCGSD, where G stands for general. This function includes all kinds of substitution operators, i.e., both ${ }^{A} t$ and ${ }^{X} t$ given in Tables I and II, so that it is wider than the conventional CCSD wave function discussed in the preceding section. The number of the variables is $M$ given by Eq. (2.4), which is larger than that of CCSD, but it is still of the order of singles and doubles.

TABLE IV. Double substitution operators of Table II applied to $|0\rangle$.

\begin{tabular}{ccccc}
\hline \hline & 1 & 2 & 3 & 4 \\
\hline $1^{\prime}$ & $a_{a}^{+} a_{c}^{+} a_{k} a_{i}|0\rangle$ & $\delta_{k l} a_{a}^{+} a_{i}|0\rangle$ & 0 & 0 \\
& & $-\delta_{i k} a_{a}^{+} a_{l}|0\rangle$ & & \\
$2^{\prime}$ & $\delta_{i j} a_{c}^{+} a_{k}|0\rangle$ & $\delta_{i j} \delta_{k l}|0\rangle$ & 0 & 0 \\
& $-\delta_{j k} a_{c}^{+} a_{i}|0\rangle$ & $-\delta_{i k} \delta_{j l}|0\rangle$ & & \\
$3^{\prime}$ & 0 & 0 & 0 & 0 \\
$4^{\prime}$ & 0 & 0 & 0 & 0 \\
\hline \hline
\end{tabular}


The CCGSD wave function has some complex structure not in common with the ordinary CCSD. First, it includes noncommuting operators, which make CCGSD somewhat complicated. Second, the linked part of the ${ }^{X_{t}}$ operator is zero, $|0\rangle$, or single excitations, as seen from Tables III and IV that summarize the GSD operators applied to $|0\rangle$. Therefore, most of the ${ }^{x} t$ part works in the form of the unlinked terms ${ }^{X} t^{A} t|0\rangle$, which has the form of the multireference coupled cluster. ${ }^{19}$

We now solve the variables $C_{p}^{r}$ and $C_{p q}^{r s}$ in CCGSD by the variational method. The $\mathrm{CC}$ expansion is given by

$$
\psi=\exp (T)|0\rangle=\left[1+T+\frac{1}{2} T^{2}+\frac{1}{3 !} T^{3}+\cdots\right]|0\rangle .
$$

Since $T$ includes the noncommuting operators, the derivative of $\psi$ with respect to $C_{p}^{r}$ and $C_{p q}^{r s}$ is written as

$$
\begin{aligned}
\frac{\partial \psi}{\partial C}= & {\left[\frac{\partial T}{\partial C}+\frac{1}{2}\left(\frac{\partial T}{\partial C} T+T \frac{\partial T}{\partial C}\right)\right.} \\
& \left.+\frac{1}{3 !}\left(\frac{\partial T}{\partial C} T^{2}+T \frac{\partial T}{\partial C} T+T^{2} \frac{\partial T}{\partial C}\right)+\cdots\right]|0\rangle,
\end{aligned}
$$

where $\partial T / \partial C$ actually stands for

$$
\frac{\partial T}{\partial C_{p}^{r}}=a_{r}^{+} a_{p}
$$

and

$$
\frac{\partial T}{\partial C_{p q}^{r s}}=a_{r}^{+} a_{s}^{+} a_{q} a_{p} .
$$

Since $T$ and $\partial T / \partial C_{p}^{r}$ do not commute, we have from Eq. (5.5)

$$
\frac{\partial \psi}{\partial C_{p}^{r}} \neq a_{r}^{+} a_{p} \psi
$$

and similarly,

$$
\frac{\partial \psi}{\partial C_{p q}^{r s}} \neq a_{r}^{+} a_{s}^{+} a_{q} a_{p} \psi .
$$

Actually, $\partial \psi / \partial C$ has some complex extra terms in addition to the right-hand sides of Eqs. (5.8) and (5.9). This means that the variational CCGSD does not satisfy the sufficient condition of the exact wave function given by Eqs. (2.8) and (2.9). Further, the variational condition [Eq. (2.6)] combined with Eqs. (5.8) and (5.9) shows that we do not have Eqs. (2.1) and (2.2). Therefore, the variational CCGSD is not exact.

Nevertheless, the CCGSD has some interesting properties as described above, so that it is worth to be studied in more detail in various situations.

\section{FULL CI WAVE FUNCTION}

Full CI wave function is exact within a limited space of reference functions, but the number of the variables, $M_{\text {full-CI }}$ given by Eq. (2.10) is tremendously large, so that a practical application is limited only to a small system. However, full $\mathrm{CI}$ is practically only one method to calculate the exact wave function and offers some useful ideas for the present study. We summarize here only some aspects of the full CI that are pertinent to the present study.

The full CI wave function is written as

$$
\begin{aligned}
\psi^{\mathrm{fci}}= & C_{0}|0\rangle+\sum_{i a} C_{i}^{a} \Phi_{i}^{a}+\sum_{i j a b} C_{i j}^{a b} \Phi_{i j}^{a b}+\sum_{i j k a b c} C_{i j k}^{a b c} \Phi_{i j k}^{a b c} \\
& +\cdots=\sum_{I} C_{I} \Phi_{I}
\end{aligned}
$$

where $\Phi_{i}^{a}, \Phi_{i j}^{a b}$, etc., denote singles, doubles, etc., excitation configurations, and the secular equation is written as

$$
\left\langle\psi^{\mathrm{fci}}|(H-E)| \Phi_{I}\right\rangle=0 .
$$

The number of the variables $\left\{C_{I}\right\}$ and the configurations $\left\{\Phi_{I}\right\}$ is $M_{\text {full - CI }}$ given by Eq. (2.10).

Since the full CI space spanned by the configurations $\left\{\Phi_{I}\right\}$ is complete, any functions like $a_{r}^{+} a_{p} \Phi_{I}$, etc., and linear combinations thereof also belong to this space. If we write such function as $\Psi_{K}$, the full CI wave function satisfies

$$
\left\langle\psi^{\mathrm{fci}}|(H-E)| \Psi_{K}\right\rangle=0,
$$

since $\Psi_{K}$ is a linear combination of $\Phi_{I}$ that satisfies Eq. (6.2). Similarly, the full CI wave function satisfies

$$
\begin{aligned}
& \left\langle\psi^{\mathrm{fci}}\left|(H-E) a_{r}^{+} a_{p}\right| \Psi_{K}\right\rangle=0, \\
& \left\langle\psi^{\mathrm{fci}}\left|(H-E) a_{r}^{+} a_{s}^{+} a_{q} a_{p}\right| \Psi_{K}\right\rangle=0, \\
& \left\langle\psi^{\mathrm{fci}}\left|(H-E) a_{s}^{+} a_{t}^{+} a_{u}^{+} a_{r} a_{q} a_{p}\right| \Psi_{K}\right\rangle=0,
\end{aligned}
$$

etc. As an extreme of the above case, full CI also satisfies

$$
\begin{aligned}
& \left\langle\psi^{\mathrm{fci}}\left|(H-E) a_{r}^{+} a_{p}\right| \psi^{\mathrm{fci}}\right\rangle=0, \\
& \left\langle\psi^{\mathrm{fci}}\left|(H-E) a_{r}^{+} a_{s}^{+} a_{q} a_{p}\right| \psi^{\mathrm{fci}}\right\rangle=0, \\
& \left\langle\psi^{\mathrm{fci}}\left|(H-E) a_{s}^{+} a_{t}^{+} a_{u}^{+} a_{r} a_{q} a_{p}\right| \psi^{\mathrm{fci}}\right\rangle=0,
\end{aligned}
$$

etc.

Comparing Eqs. (6.7) and (6.8) with Eqs. (2.1) and (2.2) of Theorem II-1, we understand that the full CI must be exact, as it is. However, here, we have further Eq. (6.9) and the higher ones, and without them we cannot determine all the coefficients involved in the full CI. This may be considered to contradict with the statement of Theorem II-1 that the set of Eqs. (2.1) and (2.2) is equivalent (in necessary and sufficient sense) to the Schrödinger equation. Actually, when the wave function involves only the variables corresponding to singles and doubles, the equations corresponding to triples and highers like Eq. (6.9), etc., are unnecessary. Thus, this contradiction originates from the ansatz of the wave function. Since the ansatz of the full CI wave function includes not only singles and doubles but also triples and higher ones, we need all the equations like Eqs. (6.4)-(6.6).

\section{A PROPOSAL OF THE EXACT WAVE FUNCTION}

As Theorem II-1 and II-2 imply that the singles and doubles description of the exact wave function should be possible, we consider in this section such a possibility. 
Based on Theorem II-1 and the discussion in the previous section, we understand that when $\psi$ and $\Phi$, satisfying the following two equations:

$$
\left\langle\psi\left|(H-E) a_{r}^{+} a_{p}\right| \Phi\right\rangle=0
$$

and

$$
\left\langle\psi\left|(H-E) a_{r}^{+} a_{s}^{+} a_{q} a_{p}\right| \Phi\right\rangle=0,
$$

become identical, i.e., $\psi=\Phi$, then $\psi$ is exact, and when $\psi$ includes only singles and doubles, Eqs. (7.1) and (7.2) are suffice to determine all the unknown variables.

We propose here a method of obtaining the exact wave function by a successive diagonalization of the matrices of the order of only singles and doubles. The first step is the CI with the general singles and doubles (CIGSD) defined by

$$
\begin{aligned}
& \psi_{1}=\left(1+T_{1}\right) \psi_{0}, \\
& T_{1}=\sum_{p r}{ }^{1} C_{p}^{r} a_{r}^{+} a_{p}+\sum_{p q r s}{ }^{1} C_{p q}^{r s} a_{r}^{+} a_{s}^{+} a_{q} a_{p},
\end{aligned}
$$

where the coefficients are calculated by Eqs. (7.1) and (7.2) with $\psi=\psi_{1}$ and $\Phi=\psi_{0}$. When $\psi_{0}=0$, ordinary CIGSD, the $x_{t}$ type operators mostly drop out because of the relations given in Tables III and IV. The second step is

$$
\begin{aligned}
& \psi_{2}=\left(1+T_{2}\right) \psi_{1}, \\
& T_{2}=\sum_{p r}{ }^{2} C_{p}^{r} a_{r}^{+} a_{p}+\sum_{p q r s}{ }^{2} C_{p q}^{r s} a_{r}^{+} a_{s}^{+} a_{q} a_{p},
\end{aligned}
$$

taking $\psi_{1}$ as a reference function, and the unknown variables are determined by Eqs. (7.1) and (7.2) with $\psi=\psi_{2}$ and $\Phi$ $=\psi_{1}$. This procedure is iterated until convergence as follows with $\psi_{n}$ being given by

$$
\psi_{n}=\left(1+T_{n}\right) \psi_{n-1},
$$

where $T_{n}$ is

$$
T_{n}=\sum_{p r}{ }^{n} C_{p}^{r} a_{r}^{+} a_{p}+\sum_{p q r s}{ }^{n} C_{p q}^{r s} a_{r}^{+} a_{s}^{+} a_{q} a_{p},
$$

and the variables in $T_{n}$ are determined by

$$
\left\langle\psi_{n}\left|(H-E) a_{r}^{+} a_{p}\right| \psi_{n-1}\right\rangle=0
$$

and

$$
\left\langle\psi_{n}\left|(H-E) a_{r}^{+} a_{s}^{+} a_{q} a_{p}\right| \psi_{n-1}\right\rangle=0 .
$$

We note that the linear expansion coefficients, ${ }^{n} C_{p}^{r}$ and ${ }^{n} C_{p q}^{r s}$, are complex in general to guarantee full freedom. When converged, $\psi_{n}$ becomes identical with $\psi_{n-1}$

$$
\psi=\psi_{n}=\psi_{n-1},
$$

and the energy is

$$
E=E_{n}=E_{n-1} \text {. }
$$

Therefore, Eqs. (7.9) and (7.10) are written as

$$
\left\langle\psi\left|(H-E) a_{r}^{+} a_{p}\right| \psi\right\rangle=0
$$

and

$$
\left\langle\psi\left|(H-E) a_{r}^{+} a_{s}^{+} a_{q} a_{p}\right| \psi\right\rangle=0,
$$

respectively, and from Theorem II-1, the solution $\psi$ is the exact wave function. We note that our wave function $\psi$ also satisfies Eqs. (2.8) and (2.9) of Theorem II-2. Since the above method is variational, the energy satisfies the upperbound nature. We call this method iterative CI (ICI) method, or more explicitly, ICIGSD method.

The wave function $\psi_{n}$ is written in a closed form as

$$
\psi_{n}=\prod_{i=1}^{n}\left(1+T_{i}\right) \psi_{0}
$$

where each $T_{i}$ is different from others and has the form of Eq. (7.8). The coefficients ${ }^{i} C_{p}^{r}$ and ${ }^{i} C_{p q}^{r s}$ are determined in each step by solving the secular equation of the size of the singles and doubles

$$
\sum_{K=1}^{M}{ }^{i} C_{K}^{*}\left\langle\Phi_{K}|(H-E)| \Phi_{L}\right\rangle=0 \quad(L=1, \ldots, M),
$$

where the asterisk implies complex conjugate and $M$ is given by Eq. (2.4). The coefficients ${ }^{i} C_{K}$ run singles and doubles coefficients $\left\{{ }^{i} C_{p}^{r},{ }^{i} C_{p q}^{r s}\right\}$ and $\Phi_{K}$ run $\left\{a_{r}^{+} a_{p} \psi_{i-1}, a_{r}^{+} a_{s}^{+} a_{q} a_{p} \psi_{i-1}\right\}$, which are singles and doubles with respect to $\psi_{i-1}$. The matrices involved in this secular equation are Hermite and its dimension is $M$, which is much smaller than $M_{\text {full-CI }}$. Equation (7.16) is just the same as Eqs. (7.9) and (7.10), replacing the index $n$ to $i$. Namely, in this method, the secular equation of the size of $M$ is successively diagonalized until convergence, instead of solving once a giant secular equation of the size of $M_{\text {full-CI }}$.

Since the size-consistency ${ }^{18}$ is a necessary condition of the exact wave function, it is interesting how the present wave function satisfies this property. The origin of the size consistency is different between full CI and CC. In the full $\mathrm{CI}$, it originates from the completeness of the configuration space and therefore costs expensively, while in the $\mathrm{CC}$, it originates from the exponential ansatz, which causes some additional calculations of the unlinked terms in comparison with CI, but is not so expensive as far as we solve it by the nonvariational method. In the proposed method, the origin of the size consistency is not like full CI, but should be similar to that of the $\mathrm{CC}$. From the requirement that the expansion of Eq. (7.15) has a structure similar to the CC expansion, the coefficients ${ }^{i} C_{p}^{r}$ and ${ }^{i} C_{p q}^{r s}$ must be able to become complex numbers.

As the proposed method is variational, the solution would approach the exact wave function asymptotically from above. Since the choice of the starting function $\psi_{0}$ is arbitrary, we may choose the CCSD as $\psi_{0}$. Such a choice would shorten the iteration times more than the choice $\psi_{0}=|0\rangle$. [For example, when $\psi_{0}=\psi_{\text {full-CI }}$, the calculation converges at once as seen from Eqs. (6.7)-(6.9).]

When converged, the aimed (ground) state is exact and the associated excited states obtained simultaneously satisfy the orthogonality and the Hamiltonian-orthogonality with the ground state, important necessary conditions for the excited states. This relation between the ground and excited states is very similar to that between the SAC and SAC-CI wave functions for the ground and excited states, respectively, for- 
mulated originally by the variational method. ${ }^{21}$ More details of the ICIGSD method will be published in the near future.

\section{CONCLUSION}

This paper presents a progress report of the author's research aiming the exact wave function within singles and doubles. A conclusion of this paper is that the singles and doubles description of the exact wave function is possible. This is a consequence of the fact that the Hamiltonian involves only one and two body operators. Theorem II- 1 is the necessary and sufficient theorem and Theorem II-2 describes a sufficient condition. In the search for the exact wave function within singles and doubles, it is very important what ansatz of the wave function do we take.

We have examined the variational exponential ansatz because Theorem II-2 implies it as a candidate of the structure of the exact wave function within singles and doubles. The variational CCS for one-particle Hamiltonian is certainly shown to be exact, while the conventional CCSD cannot be exact because first it is not variational and second the operator space is too restrictive in the sense that it does not include ${ }^{x} t$ type substitution operators. When we include both ${ }^{A} t$ and ${ }^{X} t$ type substitution operators in the coupled cluster ansatz, we obtain CCGSD (GSD stands for general singles and doubles) wave function. However, the variational CCGSD is also not exact because of the noncommuting nature of the substitution operators involved.

We then analyzed some formal aspects of the full CI wave function and summarized the properties pertinent to the present study. In Sec. VII, we have proposed an ansatz of the exact wave function that satisfies Theorems II-1 and II-2, and described the method of solution. It involves a successive solution of the secular equation of the dimensions of singles and doubles, instead of solving once a giant matrix of the dimension of $M_{\text {full-CI }}$ of the full CI method. This method is called ICIGSD method and we can calculate both ground and excited states.

\section{ACKNOWLEDGMENTS}

The author thanks Dr. M. Ehara and Mr. M. Nakata for some discussions. This study has been supported financially by the special fund from the Ministry of Education, Science, Culture, and Sports.

\section{APPENDIX}

We here derive the commutation relation between $a_{c}$, $a_{k}^{+}$, and $\exp (T)$ with $T$ being the sum of the singles and doubles of the ${ }^{A} t$ type of Tables I and II,

$$
T=\sum_{i, a} C_{i}^{a} a_{a}^{+} a_{i}+\sum_{i j, a b} C_{i j}^{a b} a_{a}^{+} a_{b}^{+} a_{j} a_{i} .
$$

First, the commutation relations of $a_{c}$ and $a_{k}^{+}$with $T$ are derived as

$$
\left[a_{c}, T\right]_{-}=\alpha_{c}
$$

where

$$
\alpha_{c}=\sum_{i} C_{i}^{c} a_{i}-2 \sum_{i j a} C_{i j}^{a c} a_{a}^{+} a_{j} a_{i}
$$

and

$$
\left[a_{k}^{+}, T\right]_{-}=\beta_{k},
$$

where

$$
\beta_{k}=-\sum_{a} C_{k}^{a} a_{a}^{+}+2 \sum_{i a b} C_{i k}^{a b} a_{a}^{+} a_{b}^{+} a_{i} .
$$

Using Eqs. (A2) and (A4) and the fact that $\alpha_{c}\left(\right.$ or $\left.\beta_{k}\right)$ and $T$ commute, we can derive

$$
\left[a_{c}, \exp (T)\right]_{-}=\alpha_{c} \exp (T)
$$

and

$$
\left[a_{k}^{+}, \exp (T)\right]_{-}=\beta_{k} \exp (T) .
$$

Applying Eqs. (A6) and (A7) to $|0\rangle$, we obtain

$$
a_{c} \psi=\alpha_{c} \psi
$$

and

$$
a_{k}^{+} \psi=\beta_{k} \psi,
$$

where $\psi$ is the CCSD wave function, $\psi=\exp (T)|0\rangle$.

${ }^{1}$ P. R. Surján, Second Quantized Approach to Quantum Chemistry; An Elementary Introduction (Springer, Berlin, 1989).

${ }^{2}$ K. Husimi, Proc. Phys. Math. Soc. Jpn. 22, 264 (1940).

${ }^{3}$ P.-O. Löwdin, Phys. Rev. 99, 1474 (1955); 99, 1490 (1955).

${ }^{4}$ E. R. Davidson, Reduced Density Matrices in Quantum Chemistry, Academic, New York (1976).

${ }^{5}$ C. Garrod and J. K. Percus, J. Math. Phys. 6, 763 (1964); C. Garrod and M. Rosina, ibid. 10, 1855 (1969).

${ }^{6}$ L. J. Kijewski, Phys. Rev. A 6, 31 (1972); 9, 2263 (1974).

${ }^{7}$ H. Nakatsuji, Phys. Rev. A 14, 41 (1976).

${ }^{8}$ H. Nakatsuji, Theor. Chem. Acc. 102, 97 (1999)

${ }^{9}$ A. J. Coleman, Rev. Mod. Phys. 35, 668 (1963).

${ }^{10} \mathrm{~F}$. Colmenero and C. Valdemoro, Int. J. Quantum Chem. 62, 369 (1994); C. Valdemoro, L. M. Tel, and E. Pérez-Romero, Adv. Quantum Chem. 28, 33 (1997).

${ }^{11}$ H. Nakatsuji and K. Yasuda, Phys. Rev. Lett. 76, 1039 (1996); K. Yasuda and H. Nakatsuji, Phys. Rev. A 56, 2648 (1997).

${ }^{12}$ D. A. Mazziotti, Phys. Rev. A 57, 4219 (1998).

${ }^{13}$ M. Nakata, M. Ehara, K. Yasuda, and H. Nakatsuji, J. Chem. Phys. 112, 8772 (2000)

${ }^{14} \mathrm{H}$. Nakatsuji, in Many-Electron Densities and Reduced Density Matrices, edited by J. Cioslowski (Kluwer Academic, New York, 2000).

${ }^{15}$ H. Nakatsuji, J. Chem. Phys. 67, 1312 (1977).

${ }^{16} \mathrm{~L}$. Pauling and E. B. Wilson, Jr., Introduction to Quantum Mechanics (McGraw-Hill, New York, 1935), p. 189.

${ }^{17} \mathrm{H}$. Nakatsuji (in preparation).

${ }^{18}$ J. A. Pople, J. S. Binkley, and R. Seeger, Int. J. Quantum Chem., Symp. 10, 1 (1976); R. J. Bartlett and G. D. Purvis III, Phys. Scr. 21, 255 (1980).

${ }^{19}$ H. Nakatsuji, J. Chem. Phys. 83, 713 (1985).

${ }^{20}$ H. Nakatsuji and K. Hirao, J. Chem. Phys. 68, 2053 (1978).

${ }^{21}$ H. Nakatsuji, Chem. Phys. Lett. 59, 362 (1978).

${ }^{22}$ H. Nakatsuji, Chem. Phys. Lett. 67, 329 (1979).

${ }^{23}$ H. Nakatsuji, in Computational Chemistry, Reviews of Current Trends; edited by J. Lesczynski (World Scientific, Singapore, 1996), Vol. 2, pp.62-124.

${ }^{24}$ W. Kutzelnigg, Theor. Chim. Acta 80, 349 (1991).

${ }^{25}$ D. J. Thouless, Nucl. Phys. 21, 225 (1960). 